BULGARIAN ACADEMY OF SCIENCES

CYBERNETICS AND INFORMATION TECHNOLOGIES • Volume 15, No 4

Sofia $\bullet 2015$

Print ISSN: 1311-9702; Online ISSN: 1314-4081

DOI: $10.1515 /$ cait-2015-0053

\title{
Robust Stability Analysis for a Perturbed Single-Area Power System Model
}

\section{Svetoslav Savov, Ivan Popchev}

Institute of Information and Communication Technologies, BAS, Sofia, Bulgaria

Email:savovsg@mail.bg

Abstract: This research work investigates the derivation of a fixed upper matrix bound for the solution of one class of parameter-dependent Continuous Algebraic Lyapunov Equations (CALE). It is supposed that the nominal coefficient matrix is subjected to real structured parametric uncertainty belonging to a convex set. The bound is used to analyze the robust stability and the performance behavior of a load-frequency control system for a single area power system model. By means of the bound one can easily estimate the distance from instability of the uncertain system and the linear quadratic performance index associated with it. The applicability of the obtained results is illustrated by an example.

Keywords: Lyapunov equation, solution bounds, uncertain systems, power systems.

\section{Introduction}

The problem of deriving bounds for the solution of the Continuous Algebraic Lyapunov Equations (CALE) has attracted the interest for more than half a century. This is due to both theoretical and practical reasons. In some cases, due to the high order, the direct solution of this equation is impossible, and in other ones, it is sufficient to have at disposal only some estimates for it. The main difficulty arises from the fact, that the available upper bounds are valid under some assumed restrictions imposed on the coefficient matrix. Due to this, valid solution bounds are possible only for some special subsets of negative stable (Hurwitz) coefficient matrices. All significant results in the area are summarized and discussed in [9].

The robustness of a linear system, subjected to structured real parametric uncertainty, belonging to some compact vector set (e.g., the unit simplex), has been recognised as a key issue in the analysis of control systems [1-6]. The main purpose of this research is to derive a fixed upper matrix bound for the solution of one class of parameter-dependent CALEs. Such bounds help to analyze the uncertain system 
with respect to stability and a quadratic performance index. A state space model with real data of a single area power system is used as a test example.

The following notations will be used: $A>0(A \geq 0)$ indicates that $A$ is a positive (semi-positive) definite matrix; $\alpha=\left(\alpha_{i}\right) \in \mathbf{R}^{N}$ denotes a real $n \times 1$ vector $\alpha$ with nonnegative entries $\alpha_{i}, i=1, \ldots, N$, and $|\alpha|$ is the sum of its entries; $A^{1 / 2}, A^{-1}, A^{\mathrm{T}}$ are the square roots (if $A$ is a positive semi-definite), the inverse (if $A$ is nonsingular) and the transpose of a matrix $A ; \lambda_{\mathrm{m}}(A), \lambda_{\mathrm{M}}(A)$ denote the minimal and maximal eigenvalue of a matrix $A$ with only real eigenvalue, respectively; the maximal singular value of an arbitrary matrix $M$ (spectral norm of $M$ ) is denoted by $\sigma_{1}(M)=\lambda_{\mathrm{M}}^{1 / 2}\left(M M^{\mathrm{T}}\right)$; the real part of the $i$-th eigenvalue of matrix $A$ is $\operatorname{Re} \lambda_{i}(A) ; v^{*}$ is the conjugate transpose of a complex vector $v$. All matrices are $n \times n$. The identity matrix is denoted as $I$. Define also the set of $n \times n$ uncertain matrix polynomials:

$$
\boldsymbol{P} \equiv\left\{A(\alpha), \quad \alpha=\left(\alpha_{i}\right) \in \mathbf{R}^{N}: \quad A(\alpha)=A+\sum_{i=1}^{N} \alpha_{i} A_{i}, \quad 0 \leq|\alpha| \leq 1\right\},
$$

where $A, A_{i}, i=1, \ldots, N$, are some fixed matrices.

\section{Preliminaries}

Consider the state space model of a linear continuous-time uncertain system:

$$
\dot{x}=A(\alpha) x, \quad x(0)=x_{0}, \quad A(\alpha) \in \boldsymbol{P},
$$

and the associated with it parameter-dependent CALE

$$
A^{\mathrm{T}}(\alpha) P(\alpha)+P(\alpha) A(\alpha)=-Q, \quad Q>0 .
$$

From Lyapunov's stability theorem it follows that if $A(\alpha)$ is a Hurwitz (negative stable) matrix for all admissible vectors $\alpha$, i.e.,

$$
s(\alpha)=-\max \operatorname{Re} \lambda_{i}[A(\alpha)]>0, \quad i=1, \ldots, n,
$$

then $P(\alpha)$ is the unique positive definite solution of (2) for any given positive definite matrix $Q$. In this case, the performance of the system can be evaluated by the index:

$$
J\left(\alpha, x_{0}\right)=\int_{0}^{\infty} x^{\mathrm{T}}(\alpha) Q x(\alpha)=x_{0}^{\mathrm{T}} P(\alpha) x_{0} .
$$

It is desired to determine parameter independent bounds for the:

(a) positive definite solution $P(\alpha)$ in (2);

(b) distance from the instability $s(\alpha)$ in (3);

(c) system performance index $J\left(\alpha, x_{0}\right)$ in (4).

Before that, the following simple results will be presented.

Lemma 1. A symmetric uncertain polynomial $X(\alpha) \in \boldsymbol{P}$ is positive definite if and only if it is positive definite at $N+1$ vertices, i.e., 


$$
X>0, \quad X+X_{i}>0, \quad i=1, \ldots, N .
$$

In this case, for the positive scalar

$$
\mu=\max \left\{\lambda_{\mathrm{M}}\left(Q X^{-1}\right), \quad \lambda_{\mathrm{M}}\left[Q\left(X+X_{i}\right)^{-1}\right], \quad i=1, \ldots, N\right\},
$$

one has

$$
\mu X(\alpha) \geq Q \quad \forall \alpha .
$$

P r o of. Suppose that $X(\alpha)$ is a positive definite polynomial for all $\alpha$. Then, the matrix inequalities in (5) must hold, by necessity, which proves the necessity part. Now, let the set of matrix inequalities (5) holds. Since the sum $|\alpha|$ of the entries of vector $\alpha$ belongs to the interval [0, 1], there always exists some nonnegative scalar $\alpha_{0}$, such that $\alpha_{0}+|\alpha|=1$. This results in $N+1$ matrix inequalities:

$$
\alpha_{0} X \geq 0, \quad \alpha_{i}\left(X+X_{i}\right) \geq 0, \quad i=1, \ldots, N,
$$

with at least one of them being strict. By summing up the left and right-hand sides one gets

$$
X+\sum_{i=1}^{N} \alpha_{i} X_{i}=X(\alpha)>0 \quad \forall \alpha .
$$

This proves the sufficiency part and completes the proof of the first statement.

Let $X(\alpha)$ be a positive definite polynomial for all $\alpha$ and consider the scalar defined in (6). Obviously, its choice guarantees that:

$$
Y=\mu X-Q \geq 0, \quad Y+Y_{i}=\mu X-Q+\mu X_{i} \geq 0, \quad i=1, \ldots, N .
$$

The application of the same arguments used to prove the first statement, results in inequality (7).

Lemma 2. Let $A(\alpha)$ be a Hurwitz matrix for all $\alpha$. If there exists a fixed symmetric matrix $P_{\mathrm{U}}$, such that

$$
A^{\mathrm{T}}(\alpha) P_{\mathrm{U}}+P_{\mathrm{U}} A(\alpha) \leq-Q \quad \forall \alpha,
$$

then $P_{\mathrm{U}}$ is an upper parameter independent matrix bound for the solution $P(\alpha)$ in (2), i.e., $P(\alpha) \leq P_{\mathrm{U}} \forall \alpha$.

P r o of. If the above inequality holds, having in mind (2), one has:

$$
A^{\mathrm{T}}(\alpha)\left[P_{\mathrm{U}}-P(\alpha)\right]+\left[P_{\mathrm{U}}-P(\alpha)\right] A(\alpha) \leq 0 \quad \forall \alpha .
$$

This is possible only if $P_{\mathrm{U}}-P(\alpha) \geq 0 \forall \alpha$, in accordance with Lyapunov's stability Theorem.

Corollary 1. If the assumptions of Lemma 2 hold, then having in mind the scalars in (3) and (4), one gets the following parameter independent bounds for the distance from instability and the performance index of the uncertain system (1):

$$
\begin{gathered}
s(\alpha) \geq s=\frac{1}{2} \lambda_{n}\left(Q P_{\mathrm{U}}^{-1}\right) \quad \forall \alpha, \\
J\left(\alpha, x_{0}\right) \leq J\left(x_{0}\right)=x_{0}^{\mathrm{T}} P_{U} x_{0} \quad \forall \alpha \text { and } \forall x_{0} .
\end{gathered}
$$

$P \quad r \quad o \quad o f$. Let $\gamma$ denotes an eigenvector of $A(\alpha)$ corresponding to the eigenvalue $\lambda$ with the largest real part for all uncertain vectors $\alpha$, i.e., 
$A(\alpha) \gamma=\lambda \gamma$. Consider the matrix inequality (8) and the associated with it scalar inequality:

$$
\begin{gathered}
\gamma^{*} Q \gamma \leq-\gamma^{*}\left[A^{\mathrm{T}}(\alpha) P_{\mathrm{U}}+P_{\mathrm{U}} A(\alpha)\right] \gamma=-\left(\lambda^{*} \gamma^{*} P_{\mathrm{U}} \gamma+\lambda \gamma^{*} P_{\mathrm{U}} \gamma\right)= \\
=-2 \operatorname{Re}(\lambda) \gamma^{*} P_{\mathrm{U}} \gamma=2 s(\alpha) \gamma^{*} P_{\mathrm{U}} \gamma .
\end{gathered}
$$

Note that $P_{\mathrm{U}}$ must be a positive definite matrix by necessity. Finally, denoting $\varphi=P_{\mathrm{U}}^{1 / 2} \gamma$ results in the inequality

$$
\lambda_{n}\left(Q P_{\mathrm{U}}^{-1}\right)=s \leq \frac{\varphi^{*} P_{\mathrm{U}}^{-1 / 2} Q P_{\mathrm{U}}^{-1 / 2} \varphi}{2 \varphi^{*} \varphi} \leq s(\alpha) .
$$

This proves the bound in (9). The upper bound (10) is obvious.

\section{The power system model}

The purpose of operating load-frequency control is to keep the frequency changes during the load sharing in some desired limits. The main change parameters of a power system are the rotor angle, the change in frequency and the active power flow between the connection lines. The linear model given below is taken from [7] and is sufficient to express the dynamic behavior of the system around the working point [8]:

$$
\begin{gathered}
\Delta \dot{P}_{\mathrm{v}}=-\frac{1}{\tau_{\mathrm{g}}} \Delta P_{\mathrm{v}}-\frac{1}{R \tau_{\mathrm{g}}} \Delta f, \\
\Delta \dot{P}_{\mathrm{m}}=\frac{1}{\tau_{\mathrm{T}}} \Delta P_{\mathrm{v}}-\frac{1}{\tau_{\mathrm{T}}} \Delta P_{\mathrm{m}}, \\
\Delta \dot{f}=\frac{1}{2 H} \Delta P_{\mathrm{m}}-\frac{D}{2 H} \Delta f-\frac{1}{2 H} \Delta P_{\mathrm{L}},
\end{gathered}
$$

where the respective parameters have the following physical meanings: $\Delta P_{\mathrm{v}}, \Delta P_{\mathrm{m}}, \Delta f$ denote the change in a turbine valve position power, turbine mechanic exit power and frequency, respectively; $\tau_{\mathrm{g}}, \tau_{\mathrm{T}}$ are the speed regulator time constant and the turbine time constant, respectively; $H$ and $D$ denote the generator inertia constant and the power system constant. The control input is $\Delta P_{\mathrm{L}}$ and denotes the load change. Using the notations

$$
x=\left(\begin{array}{lll}
\Delta P_{\mathrm{v}} & \Delta P_{\mathrm{m}} & \Delta f
\end{array}\right)^{\mathrm{T}}, \quad u=\Delta P_{\mathrm{L}},
$$

the system is put in a standard state space description of an open-loop system:

$$
\dot{x}=A_{0} x+b u, \quad A_{0}=\left[\begin{array}{ccc}
-\frac{1}{\tau_{\mathrm{g}}} & 0 & -\frac{1}{C \tau_{\mathrm{g}}} \\
\frac{1}{\tau_{\mathrm{T}}} & -\frac{1}{\tau_{\mathrm{T}}} & 0 \\
0 & \frac{1}{2 H} & -\frac{D}{2 H}
\end{array}\right], \quad b=\left(\begin{array}{c}
0 \\
0 \\
-\frac{1}{2 H}
\end{array}\right) .
$$

The following values for the parameters are used:

$$
\tau_{\mathrm{g}}=0.2 \mathrm{~s}, \tau_{\mathrm{T}}=0.5 \mathrm{~s}, C=0.05 \mathrm{pu}, H=5 \mathrm{~s}, D=0.08 .
$$


The state and control matrices are computed as:

$$
A_{0}=\left[\begin{array}{ccc}
-5 & 0 & -100 \\
2 & -2 & 0 \\
0 & 0.1 & -0.008
\end{array}\right], \quad b=\left(\begin{array}{c}
0 \\
0 \\
-0.1
\end{array}\right) .
$$

Although the open-loop system is stable, a procedure of an optimal linear quadratic regulator synthesis is suggested via the solution of the algebraic Riccati equation is as follows:

$$
\begin{aligned}
-\widetilde{Q}= & A_{0}^{\mathrm{T}} R+R A_{0}-R b b^{\mathrm{T}} R=\left(A_{0}-b b^{\mathrm{T}} R\right)^{\mathrm{T}} R+R\left(A_{0}-b b^{\mathrm{T}} R\right)+R b b^{\mathrm{T}} R= \\
& =\left(A_{0}-b K\right)^{\mathrm{T}} R+R(A-b K)+R b b^{\mathrm{T}} R=A^{\mathrm{T}} R+R A+R b b^{\mathrm{T}} R .
\end{aligned}
$$

The close loop system with a state feedback control law $u=-K x, K=b^{\mathrm{T}} R$, becomes

$$
\dot{x}=\left(A_{0}-b K\right) x=A x,
$$

where $R$ denotes the Riccati equation solution. The close loop system state matrix $A$ satisfies Lyapunov-like equation

$$
A^{\mathrm{T}} R+R A=-Q, \quad Q=R b b^{\mathrm{T}} R+\widetilde{Q} .
$$

The Riccati equation (12) has been solved for

$$
\begin{aligned}
& \widetilde{Q}=Q_{1}=\operatorname{diag}(3,10,7), \\
& \widetilde{Q}=Q_{2}=\operatorname{diag}(3,7,10), \\
& \widetilde{Q}=Q_{3}=\operatorname{diag}(7,3,10) .
\end{aligned}
$$

Respective gain matrices have been computed:

$$
\begin{gathered}
K_{1}=\left(\begin{array}{lll}
0.6323 & 0.3297 & -35.5809
\end{array}\right)=b^{\mathrm{T}} R_{1}, \\
K_{2}=\left(\begin{array}{lll}
0.5441 & 0.1205 & -33.0592
\end{array}\right)=b^{\mathrm{T}} R_{2}, \\
K_{3}=\left(\begin{array}{lll}
0.7364 & -0.3402 & -38.4281
\end{array}\right)=b^{\mathrm{T}} R_{3} .
\end{gathered}
$$

Having in mind (11) we want to investigate the robustness properties of the nominal systems

$$
\dot{x}=\left(A_{0}-b K_{s}\right) x=\bar{A}_{s} x, \quad s=1,2,3,
$$

by including the action of an additive structured polynomial perturbation, i.e.,

$$
\dot{x}=\bar{A}_{s} x+\sum_{i=1}^{N} \alpha_{i} A_{s, i} x=A_{s}(\alpha) x, \quad A_{s}(\alpha) \in \boldsymbol{P}, \quad s=1,2,3 .
$$

This puts the uncertain system in the form (1) for any given $s$. A sufficient condition for its stability is due to Lyapunov's stability theorem, which in the case when a fixed Lyapunov function is required, is given by the matrix inequality:

$$
\begin{gathered}
0>A_{s}^{\mathrm{T}}(\alpha) R_{s}+R_{s} A_{s}(\alpha)=A_{s}^{\mathrm{T}} R_{s}+R_{s} A_{s}+\sum_{i=1}^{N} \alpha_{i}\left(A_{s, i}^{\mathrm{T}} R_{s}+R_{s} A_{s, i}\right)= \\
=X_{s}+\sum_{i=1}^{N} \alpha_{i} X_{s, i}=X_{s}(\alpha) .
\end{gathered}
$$


Since $X_{s}(\alpha) \in \boldsymbol{P}, s=1,2,3$, having in mind (12), and in accordance with Lemma 1, this equality has a simple parameter independent solution:

(13) $-X_{s}=Q_{s}>0, \quad-X_{s}-X_{s, i}=Q_{s}-A_{s, i}^{\mathrm{T}} R_{s}-R_{s} A_{s, i}>0, \quad i=1, \ldots, N$, and $s=1,2,3$.

Let $N=2$ and

$$
\begin{array}{ccc}
A_{1,1} & =\left[\begin{array}{lll}
0 & 0 & 0 \\
1 & 0 & 0 \\
0 & 0 & 0
\end{array}\right], & A_{1,2}=\left[\begin{array}{lll}
0 & 0 & 1 \\
0 & 0 & 0 \\
0 & 0 & 0
\end{array}\right], \\
A_{2,1}=\left[\begin{array}{lll}
0 & 0 & 10 \\
0 & 0 & 20 \\
0 & 0 & 0
\end{array}\right], & A_{2,2}=\left[\begin{array}{lll}
0 & 0 & 20 \\
0 & 0 & 10 \\
0 & 0 & 0
\end{array}\right], \\
A_{3,1}=\left[\begin{array}{lll}
0 & 0 & 0 \\
4 & 0 & 0 \\
0 & 0 & 0
\end{array}\right], & A_{3,2}=\left[\begin{array}{lll}
0 & 0 & 1 \\
0 & 0 & 0 \\
0 & 0 & 0
\end{array}\right] .
\end{array}
$$

All matrices in (13) are positive definite, which guarantees negative definiteness of $X_{s}(\alpha)$, stability of the uncertain state matrix $A_{s}(\alpha)$ and of the system for all $\mathrm{s}=1,2,3$ and for all admissible vectors $\alpha$.

Next, the computation of the upper matrix bounds for CALEs solution will be illustrated. Let the right-hand side in the parameter-dependent CALE (2) be the identity matrix. The scalars $\mu_{s}, s=1,2,3$, in (6) have been computed as follows:

$$
\begin{aligned}
& \mu_{s}=\max \left\{\lambda_{M}\left(-X_{s}\right), \lambda_{M}\left[\left(-X_{s}-X_{s, i}\right)^{-1}\right], \quad s=1,2,3, \quad i=1,2,\right. \\
& \mu_{1}=\max \{0.2156, \quad 0.8623, \quad 0.1648\}=0.8623=\lambda_{\mathrm{M}}\left[\left(-X_{1}-X_{1,1}\right)^{-1}\right], \\
& \mu_{2}=\max \left\{\begin{array}{lll}
0.2156, & 0.3089, & 0.2154\}=0.3089=\lambda_{\mathrm{M}}\left[\left(-X_{2}-X_{2,1}\right)^{-1}\right], \\
\mu_{3}=\max \{0.2156, & 0.4178, & 0.2064\}=0.4178=\lambda_{\mathrm{M}}\left[\left(-X_{3}-X_{3,1}\right)^{-1}\right] .
\end{array}\right.
\end{aligned}
$$

According to Lemma 1, one has

$$
-I \geq \mu_{s} X_{s}(\alpha)=A_{s}^{\mathrm{T}}(\alpha)\left(\mu_{s} R_{s}\right)+\left(\mu_{s} R_{s}\right) A_{s}(\alpha)=A_{s}^{\mathrm{T}}(\alpha) P_{\mathrm{Us}}+P_{\mathrm{Us}} A_{s}(\alpha) .
$$

Due to Lemma 2, this means that

$$
\begin{aligned}
P_{\mathrm{U} 1} & =\left[\begin{array}{ccc}
0.4102 & 0.4650 & -5.4527 \\
0.4650 & 1.9902 & -2.8428 \\
-5.4527 & -2.8428 & 306.8139
\end{array}\right]=\mu_{1} R_{1}, \quad \lambda_{1}\left(P_{\mathrm{U} 1}\right)=306.9375, \\
P_{\mathrm{U} 2} & =\left[\begin{array}{ccc}
0.1323 & 0.1219 & -1.6807 \\
0.1219 & 0.5208 & -0.3723 \\
-1.6807 & -0.3723 & 102.1199
\end{array}\right]=\mu_{2} R_{2}, \quad \lambda_{1}\left(P_{\mathrm{U} 2}\right)=102.149, \\
P_{\mathrm{U} 3} & =\left[\begin{array}{ccc}
0.3008 & 0.0774 & -3.0768 \\
0.0774 & 0.3723 & 1.4215 \\
-3.0768 & 1.4215 & 160.5526
\end{array}\right]=\mu_{3} R_{3}, \quad \lambda_{1}\left(P_{\mathrm{U} 3}\right)=160.6242,
\end{aligned}
$$


are respective fixed upper matrix bounds for the parameter-dependent solution of the CALEs (2) for all $\alpha$.

From (9) one gets

$$
\begin{array}{ll}
s_{1}(\alpha) \geq s_{1}=\frac{1}{2} \lambda_{n}\left(P_{\mathrm{U} 1}^{-1}\right)=0.0016 & \forall \alpha, \\
s_{2}(\alpha) \geq s_{2}=\frac{1}{2} \lambda_{n}\left(P_{\mathrm{U} 2}^{-1}\right)=0.0049 & \forall \alpha, \\
s_{3}(\alpha) \geq s_{3}=\frac{1}{2} \lambda_{n}\left(P_{\mathrm{U} 3}^{-1}\right)=0.0031 & \forall \alpha .
\end{array}
$$

The nominal system state matrix $A_{0}$ is affected by two independent uncertain parameters, which perturb one entry each, in cases 1 and 3 . The number of perturbed entries in case 2 is two for each parameter. The maximal singular values of matrices $K_{s}$ and $A_{s, i}, s=1,2,3$, and $i=1,2$, are suitable to express the control energy expense used to ensure the respective robustness properties of the uncertain systems.

Case 1. $\sigma_{1}\left(A_{1,1}\right)=\sigma_{1}\left(A_{1,2}\right)=1, \sigma_{1}\left(K_{1}\right)=35.588, s_{1}(\alpha) \geq 0.0016$.

Case 2. $\sigma_{1}\left(A_{2,1}\right)=\sigma_{1}\left(A_{2,2}\right)=22.3607, \sigma_{1}\left(K_{2}\right)=33.0639, s_{2}(\alpha) \geq 0.0049$.

Case 3. $\sigma_{1}\left(A_{3,1}\right)=4, \sigma_{1}\left(A_{3,2}\right)=1, \sigma_{1}\left(K_{3}\right)=38.4367, s_{3}(\alpha) \geq 0.0031$.

The results obtained in case 3 show clearly that the close loop nominal system is subjected to the largest (in sense of spectral norm) perturbation, the control energy expense is the smallest one, and the distance from instability of the uncertain system is the largest in comparison with cases 1 and 3 . Also, since

$$
P_{\mathrm{U} 2}<P_{\mathrm{U} 3}<P_{\mathrm{U} 1},
$$

it follows that the upper matrix estimate $P_{\mathrm{U} 2}$ is the tightest one.

An upper estimate for the performance index in (10) can be easily computed for any given initial state vector.

Acknowledgements: The research work presented in this paper is partially supported by FP7 Grant AComIn No 316087, funded by the European Commission in Capacity Programme 2012-2016.

\section{References}

1. B 1 i m a n, P. A Convex Approach to Robust Stability for Linear Systems with Uncertain Scalar Parameters. - SIAM Journal of Control and Optimization, Vol. 42, 2004, No 6, pp. 2016-2042.

2. B 1 i m a n, P. An Existence Result for Polynomial Solutions of Parameter-Dependent LMIs. Systems and Control Letters, Vol. 51, 2004, No 3, pp. 165-169.

3. Che s i, G., A. G a r u 11 i, A. T e s i, A. V i c i n o. Polynomially Parameter-Dependent Lyapunov Functions for Robust Stability of Polytopic Systems: An LMI Approach. - IEEE Transactions on Automatic Control, Vol. 50, 2005, No 3, pp. 365-379.

4. G e ro m e 1, J., R. K or o g u i. Analysis and Synthesis of Robust Control Systems Using Linear Parameter Dependent Lyapunov Functions. - IEEE Transactions on Automatic Control, Vol. 51, 2006, No 12, pp. 1984-1988.

5. Grman, L., D. Rosinova, V. Vesely, A. Kozakova. Robust Stability Conditions for Polytopic Systems. - Journal of System Science, Vol. 36, 2005, No 15, pp. 961-973. 
6. Olive ir a, R., P. P er e s. Parameter-Dependent LMIs in Robust Analysis: Characterization of Homogeneous Polynomially Parameter-Dependent Solutions via LMI Relaxations. - IEEE Transactions on Automatic Control, Vol. 52, 2007, No 7, pp. 1334-1340.

7. Ka ki 11 i, A., Y. O g u z, H. C a 1 i k. The Modelling of Electric Power Systems on the State Space and Controlling of Optimal LQR Load-Frequency. - Journal of Electrical and Electronics Engineering, Vol. 9, 2009, No 2, pp. 977-982.

8. S a a d a t, H. Power System Analysis. New York, McGraw Hill, 1999.

9. S a v o v, S. Solution Bounds for Algebraic Equations in Control Theory. Sofia, Prof. M. Drinov Academic Publishing House, 2014. 